A. Joseph Layon MD, Stanley H. Rosenbaum MD, Lynn Dirk MAMC

\section{Human immuno-} deficiency virus and health care workers: risking livelihood as well as life

TABLE Number of HC workers with Occupationally Transmitted Human Immunodeficiency Virus from 1981 through December 1995, United States, Type of Exposure*

I N 1989, one of the first health care (HC) workers to contract the human immunodeficiency virus (HIV) from a patient alerted HC workers not to the risk of contracting HIV, but to the risk that follows it - that of losing income and livelihood. ${ }^{1}$ For example, in both the United States (US) and the United Kingdom (UK), government health agencies officially recommended that HIV-positive (HIV+) HC workers should "cease performing exposure prone procedures."2,3 Further, in 1993, the UK National Health Service mandated testing for hepatitis B of all $\mathrm{HC}$ workers involved in invasive work. ${ }^{3}$ The consequences of such policies are well illustrated by the case of a surgeon "who had hepatitis $\mathrm{B}$ and who continued working in order to support himself and his family." The moral is that the success of programmes such as employment restrictions or mandatory testing is intimately linked to their unintended consequences. This article addresses both the perceived need for testing and what should follow when the results are positive.

The risk to $\mathrm{HC}$ workers and their public response Although hospital administrators may be concerned about the public's fear of the risk of professional-topatient transmission of HIV, this risk is much lower than the reverse, because $\mathrm{HC}$ workers have a much higher likelihood of exposure through occupation ${ }^{4}$ and are unlikely to expose their body fluids to patients inadvertently. The Medical Expertise Retention Program, a medically-sponsored agency in the US with international scope, has assisted more than 200 $\mathrm{HC}$ workers who were or suspected they were HIV+ or who experienced needle sticks. ${ }^{5}$ In the United States, from 1981 to December 1995, 49 HC work-

\begin{tabular}{lc}
\hline Exposure & Number (\%) \\
\hline Percutaneous & $42(86 \%)$ \\
Mucocutaneous & $5(10.2 \%)$ \\
Percutaneous and Mucocutaneous & $1(2 \%)$ \\
Unknown & $1(2 \%)$ \\
TOTAL & 49 \\
\hline
\end{tabular}

* Most of those exposed were nurses or laboratory technicians

ers contracted HIV occupationally, ${ }^{6}$ most from percutaneous exposure ( $n=42,86 \%$ ) (Table). The rate of patient-to-professional transmission of HIV approximates $0.5 \%{ }^{7-10}$ Given the frequency of blood contamination and needle-sticks, however, the lifetime risk of occupationally-acquired HIV may be more significant than this, ${ }^{11-13}$ as only $12 \%$ of puncture injuries to surgeons are reported. ${ }^{13}$ Further, HC workers will be treating more HIV disease as numbers of the latter increase. ${ }^{14}$ Therefore, based on epidemiological data and accepted estimates, ${ }^{15}$ the lifetime risk to a $\mathrm{HC}$ worker is likely to be higher than $10 \%^{11-13}$ (Figure 1). Further, the risk of HIV infection extends to HC workers' spouses and children.

How do $\mathrm{HC}$ workers respond to this situation? In one article on patient-to-professional HIV transmission in orthopaedic surgeons, the Canadian authors stated that "hospitals should have ... disability insurance policies for surgeons who are occupationally infected with HIV." However, the article contained no discussion of such a disability programme. ${ }^{4}$ In a Swedish survey of 


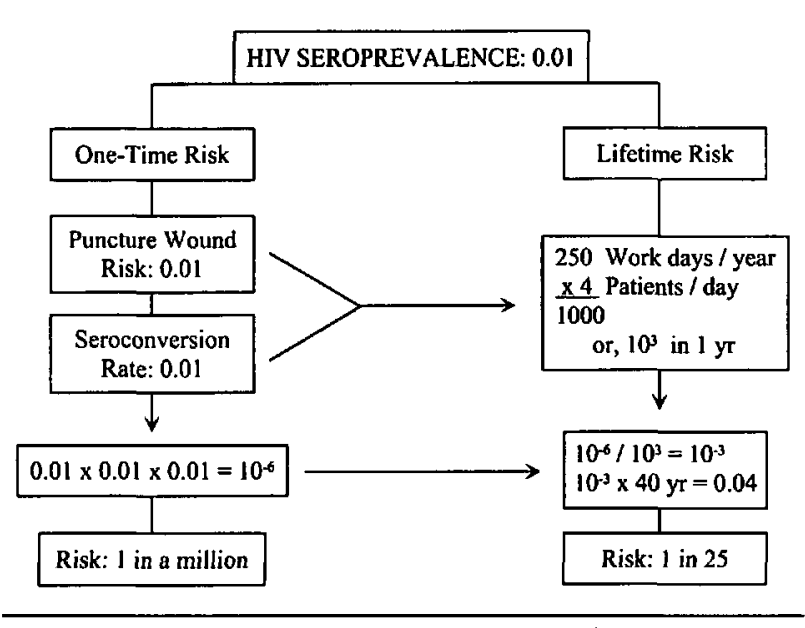

FIGURE To estimate risk of seroconversion to human immunodeficiency virus (HIV) in HC workers, assume the seroprevalence of HIV is $1 \%$ (present range is $0.02 \%$ to $5.2 \%$ ). The frequency of puncture wounds is $1 \%$ by conservative estimates and ranges as high as $2.5 \%$. After a needle-stick injury from a patient who is $\mathrm{HIV}+$, the overall risk of seroconversion is $1 \%$ (presently quored at approximately $0.5 \%$ ). An anaesthetist cares for approximately four patients per day for 250 days per year. Over a year, the risk increases to $l$ in 1000 . After a career of $40 \mathrm{yr}$, the lifetime risk of HIV transmission is approximately 1 in 25 . Even with the lower rate of seroconversion of $0.5 \%$, the risk is 1 in 50 .

$268 \mathrm{HC}$ workers on HIV-infected colleagues, $47 \%$ supported routine testing of health workers; most were opposed to disability pensioning of HIV $+\mathrm{HC}$ workers; and $73 \%$ would not tell colleagues if they became infected." A German author stated, "It is indeed high time to consider the patient HIV test as a matter of course, not least because it is our duty to be concerned also about the health of our co-workers and staff directly engaged in patient care." $t$

These reports indicate ambivalence, denial, and alarm on the part of $\mathrm{HC}$ workers who are still only at risk. The most useful reports are those by infected HC workers, like Dr. Aoun and the British surgeon who continued working illegally. Dr. Aoun summed up the situation poignantly: "What would happen to my job? How long would I live? How would my family manage? Like most physicians in training, I had no disability insurance and no life insurance." Another HIV+ surgeon said: "My experience is fairly typical of how HIV-infected people are generally treated. We are never fired because of HIV [but] because of unavoidable cutbacks, or previously undetected poor job performance... There is always a reason, and it is never the disease."2 Malpractice is another issue: after an emergency room physician admitted to his malpractice insurer that he was HIV+, his coverage was dropped and he was unable to practice. ${ }^{2}$
These reports indicate that a special form of workers' compensation or disability insurance is required for HIV+ $\mathrm{HC}$ workers. Without it, any testing will be futile and both patients and health care workers will lose. We propose a plan for HIV testing of and disability insurance for $\mathrm{HIV}+\mathrm{HC}$ workers.

\section{Testing}

Before a disability programme can be applied, occupational exposure to HIV must be established. This depends on testing, either individually after possible exposure or collectively as part of mandatory testing. Even discounting studies that have shown mandatory testing is not cost effective, the effects of such a programme could be disastrous for society and medicine ${ }^{16}$ by resulting in fraud or exorbitant costs for HIV testing and protection, or, worse, a sense of false security for the public at the expense of $\mathrm{HC}$ workers. The HC workers might refuse to treat patients at high risk of HIV infection, or who appear to be at high risk.

Individual testing after possible exposure is critical. For both social and personal reasons, $\mathrm{HC}$ workers should know their hepatitis and tuberculosis status as well as their HIV status to protect their families and themselves. Ideally, it might be sensible to test $\mathrm{HC}$ workers in a moderate- to high-risk occupation every 12 months. After exposure to a potentially HIV+ patient, both $\mathrm{HC}$ worker and patient should be tested immediately and at six weeks, three months, six months, and again at one year. ${ }^{17}$ Such surveillance is similar to that currently used for tuberculosis, varicella, and hepatitis B. Because of the social consequences of a positive result, testing should be anonymous. Insurance companies require documentation of occupational exposure, so an incident report must be filed.

An alternative is testing the patient after a $\mathrm{HC}$ worker is exposed. One system in the US gives two options ${ }^{1}$ :

- With the patient's consent, pre- and post-test counseling is offered, and the test result entered on the patient's chart.

- Without consent, such as a comatose patient without family, the test may be performed with blood obtained for another reason, and the result is not entered on the patient's chart.

With the second option, the $\mathrm{HC}$ worker is tested at the same time as the patient or must have been tested within 6 months. ${ }^{18}$ There are situations when a competent patient can refuse HIV testing. When the rights of a HC worker and a patient conflict however, who should prevail? In these cases, the HC worker is a potential patient. 


\section{Disability compensation}

For any HIV + HC worker the threat of loss of livelihood, even before loss of health, can be devastating. Therefore, the nature of the insurance is critical: with insurance as provided by hospitals to many employees, a $\mathrm{HC}$ worker may not be fully covered. If the $\mathrm{HC}$ worker cannot obtain a medical position but can perform non-medical work, disability benefits may not apply. ${ }^{19} \mathrm{~A}$ practical solution to forced unemployment and eventual disability is critical because these threats have the potential to cause $\mathrm{HC}$ workers to leave their profession en masse, to cause young people to choose occupations other than health care, or both.

We propose two options for disability protection for HC workers. The success of both depends on a pecr-based committee to determine, by sequential evaluation, when an $\mathrm{HIV}+\mathrm{HC}$ worker can no longer work in his or her usual capacity. To ensure that the interests of all parties are represented, the committee should be composed of the $\mathrm{HC}$ worker, a lay person representing patients, the pertinent department chairperson, the hospital chief-of-staff, and peer(s) of the $\mathrm{HC}$ worker, agreed on by both the infected $\mathrm{HC}$ worker and the administration.

\section{Open program}

1. A HC worker who becomes HIV+ remains in the same position. At the time of seroconversion, the committee is formed.

2. When the committee determines that the infected $\mathrm{HC}$ worker is unable to work in the same capacity, he or she, with no change in salary, will be transferred to another job within the health centre that the committee and HC worker agree is appropriate.

3. When unable to work in any capacity, disability insurance will provide a portion of the amount previously earned by the $\mathrm{HC}$ worker as salary and benefits - perhaps $80 \%$ to $90 \%$.

\section{Limited programme}

1. Health Care workers have the option to enroll in a regional or national insurance trust fund, supported by contributions from $\mathrm{HC}$ workers and employers. The $\mathrm{HC}$ worker, not enrolled in the trust fund, who becomes HIV+ is not eligible for benefits.

2. A HC worker enrolled in the trust fund who becomes HIV+:

- Remains at work under the supervision of the committee with full pay and benefits.

- Resigns at a time determined by the committee and collects salary and benefits until age 65 or 70 .
Consistency and fairness of the evaluative committee is key to the success of the programme. Neither of these proposals is radical or unworkable; they are similar to plans for impaired physicians enacted in the US in the State of Florida to be in compliance with guidelines suggested by the Centers for Disease Control. Although the proposals presented here may represent an ideal, all HC facilities must eventually address this situation.

\section{Conclusion}

At the time of the public scare over parients infected with HIV by a dentist in Florida, the resulting sense of crisis created serious division in the medical profession about the facts of HIV transmission and distortions in the public press about the frequency of transmission. ${ }^{20}$ With time, these problems have subsided, and $\mathrm{HC}$ workers now have an opportunity to reconsider how to treat HIV+ colleagues and how to educate the public. ${ }^{20}$ Whatever plan is adopted, it must address the concerns of bath patients and those who will care for them.

\section{Endnotes}

* Brattebo G, Wisborg T. HIV infection and health personnel: health care workers' opinions concerning some ethical dilemmas. [Medline abstract in English from non-English language journal] Scand J Soc Med 1990; 18: 225-9.

I Mathiak G, Wening JV, Froschle G, Junbluth KH. Is there an increased risk of infection in trauma surgery emergency admission for medical personnel by unknown HIV positive patient status? [Medline abstract in English from non-English language journal] Unfallchirurgie $1995 ; 21: 247-50$.

\section{Acknowledgments}

The authors thank James E. Thomison, Director of Legal Services, Shands Hospital at the University of Florida, and Drs. Joseph Shands, Cory Franklin, Donald Caton, and Stephen Jackson for their careful review and thoughtful comments. Anita Yeager provided invaluable editorial assistance. 
Le virus de l'immunodéficience humaine et les travailleurs sanitaires : le risque d'y perdre son gagne-pain et d'y laisser sa vie

En 1989, un des premiers cas de contamination professionnelle par un patient atteint du virus d'immunodéficience humaine (VIH) n'a pas mis les travailleurs sanitaires (TS) en garde contre le danger de contracter le VIH, mais bien contre ce qui en découle, la perte du salaire et du gagne-pain. ${ }^{1}$ Par exemple, aux EtatsUnis (É.-U.) comme au Royaume-Uni, les agences sanitaires gouvernementales recommandaient aux travailleurs sanitaires séropositifs $(\mathrm{VIH}+)$ de cesser toute activité propice à la transmission du virus. ${ }^{2,3}$ En outre, en 1993, les services de santé du Royaume-Uni.(UK National Health Service ) imposait les épreuves de détection de l'hépatite à tous les TS impliqués dans des activités effractives. ${ }^{3}$ Les conséquences de ces lignes de conduite sont bien illustrées par le cas d'un chirurgien souffrant d'hépatite $B$ qui continuait de travailler afin d'assurer sa subsistance et celle de sa famille. La morale de cette histoire est que le succès des programmes qui restreignent l'emploi ou rendent les épreuves obligatoires dépend intimement de leurs conséquences non désirées. Cet éditorial aborde à la fois le bien-fondé du dépistage et les conséquences de la positivité.

Le risque encouru par les TS et la réaction du public Bien que les administrateurs hospitaliers puissent se sentir concernés par les craintes du public devant le risque de transmission du VIH entre soignant et soigné, ce risque est plus faible que la contrepartie parce que les TS sont plus sujets à s'exposer au cours du travail ${ }^{4}$ que d'exposer leurs patients par accident à leurs liquides biologiques. Le Medical Expertise Retention Program, une agence américaine à visée internationale, a assisté plus de 200 TS qui étaient ou se croyaient séropositifs ou qui s'étaient piqués sur une aiguille. ${ }^{5}$ Aux É.-U., de 1981 à décembre 1995, 49 TS ont contracté le VIH au travail, ${ }^{6}$ la plupart par contact percutané $(\mathrm{n}=42,86 \%)$ (Tableau). Le taux de transmission du VIH de patient à
TS approchait 0,5\%. ${ }^{7-10}$ Étant donné la fréquence de la contamination sanguine et des piqûres par aiguilles, le risque à long terme de transmission professionnelle du VIH pourrait être plus élevé, ${ }^{11-13}$ seulement $12 \%$ des chirurgiens déclarant leurs lésions causées par des objets coupants. ${ }^{13}$ En outre, comme le nombre de sidatiques va en augmentant, les TS auront plus souvent l'occasion de les soigner. ${ }^{14}$ Sur la base des données épidémiologiques et des estimations reconnues, ${ }^{15}$ au cours de sa vie le TS encourt un risque de séropositivité d'au moins $10 \%{ }^{11-13}$ (Figure). Ce risque de l'infection s'étend aussi à son épouse et à ces enfants.

Comment les TS réagissent-ils à cette situation ? Dans un article canadien écrit spécifiquement pour les

TABLEAU Nombre de travailleurs sanitaires américains infectés au le travail par le VIH de 1981 à décembre 1995 et type d'exposition.

\begin{tabular}{lc}
\hline Exposition & Nombre (\%) \\
\hline Percutanée & $42(86 \%)$ \\
Mucocutanée & $5(10,2 \%)$ \\
Percutanée et mucocutanée & $1(2 \%)$ \\
Inconnue & $1(2 \%)$ \\
Total & 49 \\
\hline
\end{tabular}

*La plupart étaient des infirmiers (ères) et des techniciens de laboratoire

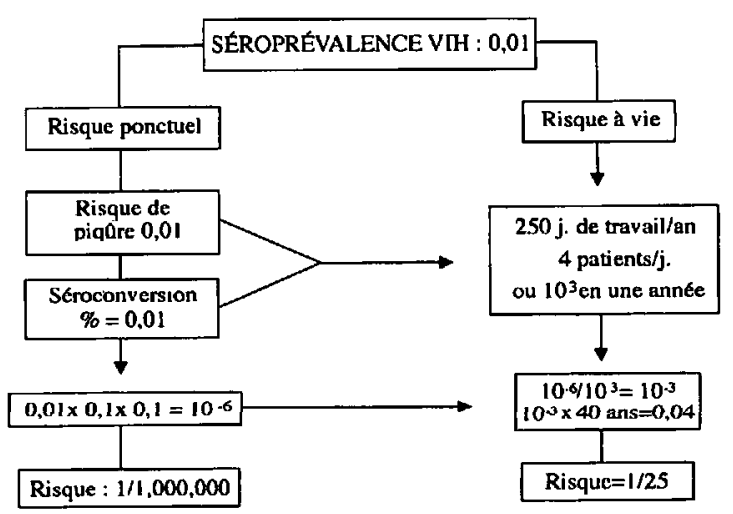

FIGURE Pour évaluer le risque de séroconversion au virus d'immunodéficience humaine (VIH) chez les travailleurs sanitaires, assumer que la séroprévalence du VIH est de $1 \%$ (présentement entre $0,02 \%$ et $5,2 \%$ ). La fréquence des blessures cutanées est de $1 \%$ d'après un estimé conservateur et peut aller jusqu'à $2,5 \%$. Après une piqûre par une aiguille contaminée par un patient $\mathrm{VIH}+$, le risque global de séroconversion est de $1 \%$ (estimé actuellement à environ $0,5 \%$ ). Un anesthésiste a la charge d'environ quatre patients par jour 250 jours par année. Sur une période d'un an, le risque augmente à 1 pour 1000. Après une carrière de 40 ans, le risque de transmission du VIH se situe appoximativement à 1 sur 25 . Même avec le taux le moins élevé de séroconcersion de $0,5 \%$, le risque se situe à 1 sur 50 . 
chirurgiens orthopédistes sur le sujet de la transmission du VIH du patient au professionnel, les auteurs énonçaient que les hôpitaux devraient avoir une politique d'assurance-invalidité destinée aux chirurgiens infectés au travail par le VIH ; cependant, ils ne spécifiaient pas les dispositions du programme. ${ }^{5}$ En Suède, au cours d'un sondage réalisé sur la question de la séropositivité de leurs collègues, $47 \%$ des 268 TS interrogés appuyaient le concept d'un test de dépistage systématique pour les TS ; la plupart s'opposaient à un programme de pension d'invalidité pour les travailleurs séropositifs ; $75 \%$ ne l'avoueraient pas à leurs collègues s'ils devenaient infectés."

Un responsable allemand déclarait qu'il était grandement temps de considérer comme inévitable pour le patient le test contre le VIH, surtout parce que le TS a le devoir de protéger la santé de ses collègues et celle du personnel directement engagé dans les soins. ${ }^{2}$ Un responsable allemand déclarait qu'il était grandement temps de considérer comme inévitable pour le patient le test contre le VIH, surtout parce que le TS a le devoir de protéger la santé de ses collègues et celle du personnel directement engagé dans les soins.

Ces rapports montrent l'ambivalence, la dénégation et l'appréhension de TS de ceux qui ne se sentent que menacés. Des comptes rendus plus alarmants nous parviennent de TS infectés, comme le Dr Aoun et ce chirurgien britannique qui continuait à travailler illégalement. Le Dr Aoun résume pathétiquement la situation : "Que va-t-il advenir de mon travail ? Pendant combien de temps vivrai-je encore ? Qu'arrivera-t-il à ma famille ? Comme la plupart des médecins en formation, je n'avais pas d'assurance-invalidité ni d'assurance-vie».' Un autre chirurgien séropositif disait : «Mon expérience est typique de la façon dont on traite généralement les gens infectés par le VIH. Nous ne sommes jamais congédiés à cause du SIDA mais à cause de coupures budgétaires inévitables, ou d'un rendement médiocre non signalé auparavant... On trouve toujours un motif mais ce n'est jamais celui de la maladie» ${ }^{2}$ Le problème causé par la responsabilité professionnelle entre aussi en jeu: après avoir avoué à son assurance médicale sa séropositivité, la protection professionnelle d'un urgentologue lui a été retirée et il lui est devenu impossible d'exercer sa profession.

Ces comptes rendus démontrent qu'une forme spéciale d'assurance contre les maladies professionnelles ou une assurance-invalidité est nécessaire pour les travailleurs sanitaires VIH positif. Sans elles, toute épreuve de dépistage devient futile et tous sont perdants, les travailleurs comme les bénéficiaires. Nous proposerons donc un plan pour le dépistage du VIH et une assurance-invalidité pour les TS séropositifs.

\section{Le dépistage}

Avant d'appliquer un programme d'assurance-invalidité, il faut établir l'incidence de l'exposition au VIH pendant le travail. Elle repose sur le dépistage, soit individuel après une exposition appréhendée, soit collectif comme élément du dépistage obligatoire. Même en écartant les études qui ont montré que les épreuves de dépistage obligatoire manquent d'efficience, les répercussions du dépistage forcé pourraient être désastreuses pour la société et la médecine ${ }^{16}$ en favorisant la fraude et en engendrant des coûts exorbitants pour le dépistage et la protection anti-VIH ou, pire encore, en propageant dans le public une impresion de fausse sécurité au dépend des TS. Les travailleurs sanitaires pourraient éventuellement refuser de prendre soin des patients à risque élevé ou qu'ils croient l'être.

Après une exposition appréhendée, le test anti-VIH devient une mesure capitale. À la fois pour des raisons sociales et personnelles, les travailleurs sanitaires doivent savoir s'ils sont atteints de la tuberculose ou de l'hépatite ou infectés par le VIH pour leur propre protection ainsi que pour celle de leur famille. Idéalement, ceux qui travaillent dans des conditions de risque modéré ou élevé devraient subir le test VIH chaque année. Après un contact avec un sujet potentiellement séropositif, le travailleur et le patient devraient subir l'épreuve immédiatement et six semaines, trois mois, six mois et une année plus tard. ${ }^{17}$ Cette surveillance est identique à celle qui s'applique couramment à la tuberculose, la varicelle et à l'hépatite B. À cause des conséquences sociales d'un résultat positif, le test devrait se faire sous le couvert de l'anonymat. Comme les compagnies d'assurance exigent une documentation de l'exposition en milieu de travail, le travailleur complétera une formule de déclaration d'accident.

Une autre solution consiste à faire subir le test au patient auquel a été exposé le travailleur sanitaire :

- Avec son consentement, on propose au patient une orientation pré- et post-test, et on inscrit au dossier le résultat de l'épreuve.

- Sans consentement, par ex. chez un comateux sans famille connue, le test est réalisé au moment d'une prise de sang pour une autre raison, et le résultat n'est pas consigné au dossier.

Avec la deuxième option, le travailleur de la santé subit le test en même temps que le patient ou avant six mois. ${ }^{18}$ Il est possible qu'un patient mentalement compétent refuse le test. Quand les droits du travailleur sanitaire et ceux du patient entrent en conflit, lesquels doivent prévaloir? Quelle que soit la situation, il ne faut jamais oublier que le travailleur devient un patient potentiel. 


\section{La compensation pour invalidité}

Pour tous les travailleurs sanitaires, même avant la perte de la santé, la menace d'une privation des moyens de subsistance peut avoir des effets dévastateurs. C'est ici que la qualité de l'assurance prend une importance primordiale ; l'assurance contribuée par l'hôpital peut être insuffisante pour son employé. Si le professionnel ne peut occuper un poste médical tout en étant apte à des activités dans un autre domaine, il est possible qu'on lui refuse l'accès aux bénéfices de l'assurance-invalidité. ${ }^{19} \mathrm{Il}$ faut à tout prix trouver des solutions aux problèmes causés par le congédiement et l'invalidité parce qu'ils peuvent provoquer des démissions massives, la désertion des soins de santé comme choix de carrière par les jeunes ou ces deux calamités à la fois.

Comme protection contre l'invalidité chez les TS, nous proposons deux options. Leur succès dépend d'un comité formé par les pairs qui déterminera après évaluation quand un TS séropositif ne peut plus fournir son rendement habituel. Pour s'assurer que tous les intérêts des parties sont représentées, le comité devrait inclure le TS, un représentant non médical des patients, le directeur du département concerné, le directeur médical et les pairs du travailleur sanitaire qu'il choisira en accord et avec les administrateurs.

\section{Programme universel}

1. Le travailleur qui devient séropositif garde son emploi. Au moment de la séroconversion, on forme le comité.

2. Quand le comité a déterminé que le travailleur infecté n'est plus capable de fournir son rendement habituel, il ne subit aucune perte de salaire et il est transféré dans le même centre hospitalier à un nouveau poste agréé par le comité et le travailleur.

3. Lorsqu'il devient tout à fait incapable de travailler, l'assurance invalidité lui fournira une portion de sa rémunération antérieure en salaire et bénéfices d'appoint - possiblement de 80 à $90 \%$.

\section{Programme limité}

1. Les travailleurs sanitaires ont l'option de joindre une société fiduciaire d'assurances régionale ou nationale supportée par les contributions des travailleurs et des employeurs. Le travailleur non enregistré avec cette fiducie devenu séropositif n'est plus éligible.

2. Le travailleur sanitaire enregistré dans ce programme devenu séropositif :

- demeure au travail sous la surveillance du comité avec pleins salaire et bénéfices
- démissionne à un moment déterminé par le comité et recueille salaire et bénéfices jusqu'à l'âge de 65 ou 70 ans.

La consistance et l'équité du comité d'évaluation constituent la clé du succès du programme. Aucune de ces propositions n'est radicale ou inapplicable ; elles sont identiques aux programmes adaptés aux médecins inaptes en vigueur aux É.-U. dans l'état de la Floride et conformes aux lignes de conduite élaborées par le Centre for Disease Control. Bien que ce qui est proposé ici représente un idéal, tous les centres hospitaliers doivent éventuellement s'attaquer à ce problème.

\section{Conclusion}

En Floride, la transmission du VIH d'un dentiste à ses patients a créé tout un émoi dans le public. La crise qui a suivi a révélé au sein de la profession médicale le profond désaccord existant sur la transmission du VIH qui fut amplifié par les inexactitudes sur la fréquence de cette transmission propagées par la presse. ${ }^{20}$ Avec le temps, la poussière $s^{\prime}$ est dispersée, et les travailleurs sanitaires ont maintenant l'opportunité d'examiner à nouveau comment il doivent traiter leurs collègues séropositifs et éduquer le public. ${ }^{20}$ Quel que soit le plan adopté, il doit s'attaquer à la fois aux préoccupations des patients et à celles de ceux en ont soin.

\section{Renvois}

* Brattebo G. Wisborg T. HIV infection and health personnel; health care workers' opinions concerning some ethical dilemmas. [Résumé anglais d'un article extrait d'un journal publié dansune autre langue] Scand J Soc Med 1990; 18; 225-9.

$\dagger$ Mathiak G, Wening JV, Froschle G, Junbluth $K H$. Is there an increased risk of infection in trauma surgery emergency admission for medical by unknown HIV positive patient status. [Résumé anglais d'un article extrait d'un journal publié dansune autre langue] Unfallchirurgie $1995 ; 21$ 247-50.

\section{Remerciements}

Les auteurs remercient James E. Thomison, directeur des services légaux, Wands Hospital, l'université de la Floride, et les Drs Joseph Shands, Cory Franklin, Donald Caton et Stephen Jackson pour leur révision et leurs judicieux commentaires. Anita Eager nous a aussi été d'un précieux secours.

\section{References}

1 Aoun $H$. When a house officer gets AIDS. N Engl J Med 1989; 321: 693-6.

2 Scoles $P$. The personal impact. Bull NY Acad Med 1995; 72(Suppl): 226-39. 
3 Newell A, Barton SE. Testing healthcare staff for infection with HIV and hepatitis: logistic and ethical considerations. J Clin Pathol 1995; 48: 885-9.

4 Wright JG, McGeer $A$. Human immunodeficiency virus transmission between surgeons and patients in orthopaedic surgery. Clin Orthop 1993; (297): 272-81.

5 Schatz B. Supporting and advocating for HIV-positive health care workers. Bull NY Acad Med 1995; 72(Suppl): 263-72.

6 Centers for Disease Control and Prevention. HIVD/AIDS Surveillance Report 1995; 7(\#2): 21.

7 Lifson $A R$, Castro KG, McCray E, Jaffe $H W$. National surveillance of AIDS in health care workers. JAMA 1986; 256: 3231-4.

$8 \mathrm{McCray}$ E. Occupational risk of the acquired immunodeficiency syndrome among health care workers. N Engl J Med 1986; 314: 1127-32.

9 Update: acquired immunodeficiency syndrome and human immunodeficiency virus infection among health care workers. MMWR 1988; 37: 229-39.

10 Henderson DK, Fabey BJ, Willy $M$, et al. Risk for occupational transmission of human immunodeficiency virus type 1 (HIV-1) associated with clinical exposures. A prospective evaluation. Ann Intern Med 1990; 113: 740-6.

11 Jones $M E$. A thing about AIDS. Anaesth Intensive Care $1989 ; 17: 253-63$.

12 McKinney WP, Young MJ. The cumulative probability of occupationally-acquired HIV infection - the risks of repeated exposures during a surgical career. Infect Control Hosp Epidemiol 1990; 11: 243-7.

13 Lowenfels $A B$, Wormser $G P$, Jain $R$. Frequency of puncture injuries in surgeons and estimated risk of HIV infection. Arch Surg 1989; 124: 1284-6.

14 Centers for Disease Control and Prevention. Projections of the number of persons diagnosed with AIDS and the number of immunosuppressed HIVinfected persons, United States, 1992-1994. MMWR 1992; 41(\#RR-18): 1-29.

15 Howard RJ. Human immunodeficiency virus testing and the risk to the surgeon of acquiring HIV. Surg Gynecol Obstet 1990; 171: 22-6.

16 Daniels $N$. HIV-infected professionals, patient rights, and the "switching dilemma." JAMA 1992; 267: 1368-71.

17 Tokars JI, Marcus R, Culver DH, et al. Surveillance of HIV infection and zidovudine use among health care workers after occupational exposure to HIV-infected blood. Ann Intern Med 1993; 118: 913-9.

18381.004 Florida Statutes (1992 Supp).

19 Lavely $R$. The HIV-positive physician and disability insurance. Ann Emerg Med 1994; 23: 1355-62.

20 Burris $S$. Human immunodeficiency virus-infected health care workers: the restoration of professional authority. Arch Fam Med 1996; 5: 102-6. 